\title{
Interactions among predators and plant specificity protect herbivores from top predators
}

\author{
Christopher Bosc (iD,${ }^{1,4}$ Francois Roets, ${ }^{2}$ Cang Hui, ${ }^{3}$ and Anton Pauw ${ }^{1}$ \\ ${ }^{1}$ Department of Botany and Zoology, Stellenbosch University, Matieland 7602 South Africa \\ ${ }^{2}$ Department of Conservation Ecology and Entomology, Stellenbosch University, Matieland 7602 South Africa \\ ${ }^{3}$ Centre for Invasion Biology, Department of Mathematical Sciences, Stellenbosch University, Matieland 7602 South Africa
}

\begin{abstract}
The worldwide loss of top predators from natural and agricultural systems has heightened the need to understand how important they are in controlling herbivore abundance. The effect of top predators on herbivore species is likely to depend on (1) the importance of the consumption of intermediate predators by top predators (intra-guild predation; IGP), but also on (2) plant specificity by herbivores, because specialists may defend themselves better (enemy-free space; EFS). Insectivorous birds, as top predators, are generally known to effectively control herbivorous insects, despite also consuming intermediate predators such as spiders, but how this effect varies among herbivore species in relation to the cascading effects of IGP and EFS is not known. To explore this, we excluded birds from natural fynbos vegetation in South Africa using large netted cages and recorded changes in abundance relative to control plots for 199 plant-dwelling intermediate predator and 341 herbivore morpho-species that varied in their estimated plant specificity. We found a strong negative effect of birds on the total abundance of all intermediate predators, with especially clear effects on spiders (strong IGP). In contrast with previous studies, which document a negative effect of birds on herbivores, we found an overall neutral effect of birds on herbivore abundance, but the effect varied among species: some species were negatively affected by birds, suggesting that they were mainly consumed by birds, whereas others, likely released from spiders by IGP, were positively affected. Some species were also effectively neutrally affected by birds. These tended to be more specialized to plants compared to the other species, which may imply that some plant specialists benefited from protection provided by EFS from both birds and spiders. These results suggest that the response of herbivore species to top predators may depend on cascading effects of interactions among predators and on their degree of plant specificity.
\end{abstract}

Key words: arthropods; birds; cascading effect; enemy-free space; herbivores; intra-guild predation; plant specificity.

\section{INTRODUCTION}

One of the most debated questions in community ecology concerns the control of herbivore populations (Hairston et al. 1960, Pace et al. 1999, Borer et al. 2005). Predators can have a major impact on herbivores (top-down control; Estes et al. 2011), notably terrestrial herbivorous insects (Vidal and Murphy 2018). However, increasing evidence suggests that interactions among predators can ameliorate the impact of predation on herbivores (Polis and Holt 1992, Vance-Chalcraft et al. 2007, Law and Rosenheim 2011, Grass et al. 2017), while specific interactions with hostplants can further mitigate the pressure of generalist predators (positive bottom-up effect) (Bernays 1989, Dyer 1995, Vencl et al. 2005, Singer et al. 2014).

Generalist top predators such as insectivorous birds exert important top-down effects on both herbivorous insects and intermediate predators such as spiders (Mooney et al. 2010, Mäntylä et al. 2011). As birds and intermediate predators often compete for the same prey, intraguild predation of birds on intermediate predators (IGP) can have indirect consequences for herbivore populations (Polis and Holt 1992). For

Manuscript received 15 December 2017; revised 21 March 2018; accepted 5 April 2018. Corresponding Editor: Jay A. Rosenheim.

${ }^{4}$ E-mail: christopherbosc@gmail.com instance, if intermediate predators are at least as good as birds at exploiting available prey, then IGP would release these prey populations (Polis and Holt 1992). Though there is ample evidence for the beneficial effect of IGP on herbivores in simple arthropod assemblages (meta-analysis: VanceChalcraft et al. 2007), this effect has less often been reported in real communities including birds as top predators (Mooney et al. 2010). The few existing cases imply herbivores mainly consumed by intermediate predators rather than birds (Martin et al. 2013, Grass et al. 2017). Even when prey is potentially shared with intermediate predators, most studies conclude that birds generally suppress both herbivore populations and intermediate predators simultaneously, i.e., herbivores derive little benefit from the effect of birds on intermediate predators (reviewed by Mooney et al. 2010).

Predation pressure on herbivores can be mitigated by enemy avoidance (Greeney et al. 2012), which can indirectly be driven by the interactions of herbivores with their host plants (bottom-up effect). The enemy-free space hypothesis (EFS) predicts that plant specificity can protect herbivores against generalist natural enemies through various mechanisms (Bernays and Graham 1988, Bernays 1989, Singer et al. 2014). For instance, plant-specialized herbivores can accumulate plant chemical compounds that are toxic for these natural enemies (Dyer 1995, Vencl et al. 2005). Also, the most plant-specialized herbivores can avoid predation 
by better matching their environment (e.g., cryptic coloration; Bernays and Graham 1988, Singer et al. 2014) or signal their potential unpalatability (aposematism; Bernays 1989, Singer et al. 2014). Recent evidence indicates that plant-specialized caterpillars better avoid predation by birds compared to generalist species and this could be explained by an association between high specificity and greater crypsis and aposematism, validating the EFS hypothesis (Singer et al. 2014). Indeed, crypsis and aposematism should be particularly effective defenses against birds because they use vision to locate prey. But plant specialists could also be protected against intermediate predators such as spiders which use tactile cues or webs, because herbivore specificity is also often associated with higher toxicity and/or lower mobility (Bernays 1989, Dyer 1995). However, the EFS hypothesis has mostly been tested with predatory insects (e.g., wasps, ants and bugs) and only once with birds (Singer et al. 2014).

Here we experimentally determined the top-down effect of birds on plant-dwelling intermediate predator (IGP) and herbivore communities dominated by spiders and hemipterans, using bird exclosures whilst simultaneously investigating the possible mitigating effect of herbivore-plant specificity (EFS; positive bottom-up effect). As studies in general have shown that both intermediate predator and herbivore communities are controlled by birds (Mooney et al. 2010), our main hypotheses and predictions concerning the overall effect of birds were that, (1) birds should control intermediate predators (IGP: intraguild predation) and (2) birds should also control overall herbivore abundance. (3) However, the effect of birds on each herbivore species should depend on cascading effects of IGP on herbivores and on the plant specificity of herbivores (enemy-free space; EFS; Singer et al. 2014). Notably, IGP should release plantgeneralist species mainly consumed by intermediate predators, while EFS should protect plant specialists from birds and from intermediate predators.

\section{Methods \\ Study site}

The study was conducted in the Jonkershoek Valley $\left(33^{\circ} 57^{\prime} \mathrm{S}\right.$ and $\left.18^{\circ} 55^{\prime} \mathrm{E}\right)$, in the Jonkershoek nature reserve (Cape Floristic Region; Western Cape; South Africa) characterized by granite and sediment soils. Insectivorous birds observed on the study sites belonged mainly to Promeropidae and Nectariniidae, whose breeding seasons, and predation, peak between May and September (Fraser 1989, Botha 2017). Bird exclosures were built between August 2013 and March 2014 in the sixth year of post-fire succession and maintained until March 2015, therefore covering the entire breading season of birds. The experiment was replicated at six different sites, three on the north facing slope and three on the south facing slope (Appendix S4: Fig. S1). The vegetation at all sites is fynbos, the dominant vegetation type in the region, characterized by shrubs and sclerophyllous plants.

\section{Experimental design}

At each site, a large exclosure cage of $20 \times 20 \times 2.2 \mathrm{~m}$ (hereafter "exclosure"), an open control plot of $20 \times 20 \mathrm{~m}$ (hereafter "open") and two $7 \times 10 \mathrm{~m}$ shaded control plots (hereafter "shade") were established (Appendix S4: Plate $\mathrm{S} 1)$. Shade plots had roofs (2.2 $\mathrm{m}$ high) but unlike the exclosure cages, did not have walls. Therefore, exclosures excluded birds from plots whereas shade plots allowed the movement of birds beneath roofs whilst controlling for the shading effect. Roofs of shade plots were smaller in area than the exclosure cages to allow birds to pass beneath them more easily. Exclosure cages and roofs consisted of fish nets (100\% high density polyethylene) fixed onto poles. Net mesh-size was $2 \times 2 \mathrm{~cm}$, which is small enough to exclude birds, but large enough to allow free movement of most arthropods. Placement of exclosures, open and shade plots varied between sites as plots were chosen to maximize plant community similarity between treatments. However, because of the high fine-scale spatial turnover of plant species in fynbos vegetation (Goldblatt and Manning 2002), it was not possible to obtain exact similarity between treatments and we therefore considered differences in plant species composition between treatments in our analyses.

Plant surveys and arthropod collections were conducted between October and December 2014, at the end of bird breeding seasons. Within each treatment and each site, eight $2.5 \times 2.5 \mathrm{~m}$ quadrats were randomly chosen (quadrat number and size were selected as a compromise between replication and sampling effort). In each quadrat, all plants were identified to the morpho-species level and the cover of each species was estimated $\left(\mathrm{m}^{2}\right)$. All arthropods present on the vegetation were sampled with the help of a vacuum sampler (modified leaf shredder vacuum/ blower STIHL SH-86d; maximum airflow: $770 \mathrm{~m}^{3} / \mathrm{h}$ ) as this method provides optimal plant-associated arthropod catches in fynbos vegetation (Swart et al. 2017). Arthropods were collected once in each quadrat and only on windless, sunny and cloudless days. In each site, quadrats were alternatively sampled in each treatment until all chosen quadrats in all treatments were sampled. The mouth of the nozzle of the vacuum sampler was applied over the vegetation by 'stabbing' at full power for 5 min, making sure to collect from all living plant parts within each quadrat. Catches per quadrat were placed into separate Ziploc bags, frozen and then preserved in $70 \%$ ethanol. All arthropod individuals were sorted to morpho-species and the number of individuals counted. Plant and arthropod morpho-species were photographed and identified to the lowest taxonomic level possible (mainly genus or species level for plants and spiders; family level for insects) with the help of identification keys for insect families (Scholtz and Holm 1985) and plants (Manning 2007) or professional taxonomists for some plants, spiders (Araneae) and Cicadellidae (Hemiptera). Each arthropod morpho-species was assigned to a trophic guild according to the dominant feeding behavior of that particular family/ subfamily as indicated by their mouthparts and published literature (Scholtz and Holm 1985). Intermediate predators included all arthropod morpho-species feeding predominantly on other arthropods by predation. Herbivores included all arthropod morpho-species that feed on leaves, stems, flowers, pollen and seeds (i.e., all arthropod taxa that may negatively affect plants through consumption). All morpho-species collected in this study are stored at the 
Stellenbosch University and photographs are provided in Dryad (see Data Availability).

\section{Analyses}

Effects of birds on total abundance of arthropods. - In order to test the overall effect of birds on intermediate predators and herbivores, we compared total intermediate predator and herbivore abundance between treatments (exclosure vs. open vs. shade). We used linear mixed models (LMMs) to estimate the differences between treatments for arthropods, while controlling for differences between sites and slopes (package lme4 in R software) (Bates et al. 2014, R Core Team 2017). Thus, in the models, we considered treatment as fixed factor and site as random factor in the form of random intercepts with fixed means which accounted for pseudo-replication due to block structure. Slope was not included as a random factor in the analyses, as its effect is masked by differences between sites. Significance of the fixed factors were determined with REML $t$-tests and Satterthwaite's approximation on the intercept in each model (package lmerTest in R software) (Kuznetsova et al. 2016, R Core Team 2017).

Effects of birds on arthropod species composition.-To test our hypotheses concerning the differential impact of birds on herbivore species in relation to IGP and plant specificity, we firstly needed to enumerate the effect of birds on each arthropod species. For that, we used a multivariate analysis, which allowed us to determine the effect size for all species at once, while accounting for plant effect. Prior to the analysis, the plant and arthropod datasets (i.e., covers or abundances per species in each quadrat) were transformed and standardized using square root transformations followed by Hellinger standardizations (Legendre and Gallagher 2001). We used partial redundancy analysis (pRDA) to detect differences in arthropod species composition as explained by differences between treatments (exclosure vs. open vs. shade), while partialling out the variance explained by plant species composition and differences between sites (and slopes) (i.e., spatial block structure) (Borcard et al. 1992). In this analysis, treatment was set as predictor, and plant species and site as conditions. As many plant species were considered $(n=125)$, we performed an orthogonalisation of the variables to alleviate potential collinearity, and used a selection of variables (see method details in Appendix S1). The pRDA was standardized, so that the variances of abundant and rare species were equal (pRDA on correlation matrix; all species were scaled to unit variance; Borcard et al. 2011). Significance was tested with ANOVA with permutations, using the sites as blocking factor (R-package: vegan) (Oksanen et al. 2017, R Core Team 2017).

Using the pRDA scores, we calculated a unique score named "B-score" ( $\mathrm{B}=$ bird) for each arthropod species based on the effect of birds on their abundance. Each score was a function of the projection of the focal arthropod coordinates in the ordination space on a "bird effect axis" that passes through the exclosure centroid and the origin of the constrained axes (Appendix S4: Fig. S2). In particular, we considered the ordination space as a Cartesian coordinate system, with the absolute B-scores obtained for each arthropod species by calculating the norm of its projected vector along the bird effect axis. Deviation of species B-scores from zero were determined with one-sample $t$-tests after testing for normality for each trophic group. Species were given negative/positive B-scores when negatively/positively affected by birds. The aim here was to distinguish the arthropod species according to the effect of birds on them: negative, neutral or positive. Specifically, arthropod species with intermediate B-scores (between $37.5 \%$ and $62.5 \%$ quartiles) were considered neutrally affected by bird exclosure. In relation to our hypotheses, arthropods with negative scores (those $<37.5 \%$ quartile) were interpreted as mainly affected by birds, with low cascading effect of IGP, and arthropods with positive scores (those $>62.5 \%$ quartile) were interpreted as mainly affected by intermediate predators, with high cascading effect of IGP. Arthropods with neutral B-scores could be interpreted as either benefiting from moderate cascading effect of IGP or be unaffected by birds and intermediate predators. In effect, these two scenarios cannot be distinguished, but a greater plant specificity of these species would support the hypothesis of predator avoidance because of EFS provided by plants. Therefore, we also estimated the specificity of arthropods to plants and how it was related to bird effect.

Estimation of plant specificity. - The classical method for determining arthropod-plant specificity (i.e., diet breath) is to sample arthropods on each plant species separately, then determine the average number of host-plant species per arthropod species (Novotný et al. 2002, Dyer et al. 2007, Singer et al. 2014). However, since, in mature communities, Fynbos plants grow intertwined and cannot be separately sampled, we used co-occurrence data of arthropod and plant species in replicated quadrats to estimate the arthropod-plant specificity. The idea is that the strength of the response of arthropods to changes in plant community composition is related to their degree of plant specificity (Kemp et al. 2017). Since very little is known about the feeding behavior of the herbivorous arthropods in the region, plant and herbivore associations were based solely on this analysis.

We used a second partial redundancy analysis (pRDA) to quantify the degree of association between each arthropod species and plant community composition (i.e., plant specificity), while controlling for spatial (block) structure and bird experiment effects. Plant species were set as predictors, and treatment and site as conditions in the analysis. The pRDA was standardized, so that the variance of each arthropod species equaled one (pRDA on correlation matrix; Borcard et al. 2011). Plant species were transformed into orthogonal principal components to avoid potential correlation, with selected principal components used in the pRDA (Appendix S1). The significance of each constrained component was tested by ANOVA with permutations, with only significant axes interpreted. All the ANOVAs were performed with a permutational block structure, using the interaction between treatment and site as the blocking factor (R-package: vegan) (Oksanen et al. 2017, R Core Team 2017).

A unique score was obtained for each arthropod species (named "P-score" for Plant-score), corresponding to the geometrical distance from the species coordinates on the constrained axes to the origin. These P-scores were interpreted as rough estimates of plant specificity for each 
arthropod species (the lowest P-scores representing plant generalists and the highest P-scores plant specialists). Then, we determined how the effect of birds on arthropods was related to their host-plant specificity by analyzing the Bscores as a function of P-scores. Specifically, the differences in P-scores between the herbivore species negatively, neutrally and positively affected by birds were determined with a LMM (Bates et al. 2014, R Core Team 2017) controlling for phylogenetic structure by using order as a random factor, and family within order as a nested random factor.

\section{RESULTS}

We collected 199 intermediate predator and 341 herbivore morpho-species, representing 12,816 individuals. $75 \%$ of intermediate predators were spiders, and the Theridiidae, Philodromidae, Clubionidae and Araneae were the most abundant (Appendix S4: Fig. S3). The Homoptera, and specifically the Cicadellidae ( $40 \%$ of all herbivores in term of abundance), was the dominant herbivore group in our samples (Appendix S4: Fig. S4). Plant communities were represented by 125 morpho-species belonging to 39 families, dominated by Proteaceae and Asteraceae in terms of species richness and abundance (Appendix S4: Fig. S5).

\section{Effects of birds on total abundance of arthropods}

In terms of total abundance per quadrat (Fig. 1; Appendix S3: Table S1), intermediate predators were significantly more abundant in the exclosure treatments (estimated mean number of individuals \pm SE: $27.9 \pm 5.2$ ) compared to both open $(14.2 \pm 2.6)$ and shade $(16.4 \pm 3.2)$ treatments which were not significantly different from each other, indicating a

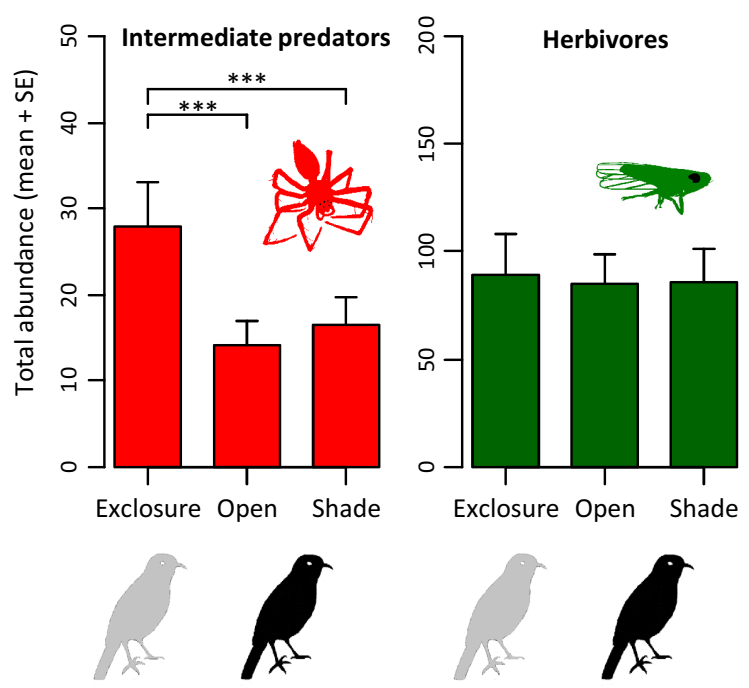

FIG. 1. Total abundances of arthropods per quadrat in each treatment for intermediate predators and herbivores: bird exclosure and open treatment were represented by 48 quadrats each, and shade treatment by 24 quadrats. Bars represent the estimated mean total abundance per treatments + standard errors determined by the linear mixed models (LMMs). Horizontal bars show the significance of the REML $t$-tests with Satterthwaite's approximation in the LMM: $* * * P \leq 0.001$. Only significant $(P \leq 0.05)$ pairwise comparisons are shown. bird effect with negligible shade effect. However, only spiders were affected and not predatory insects (Appendix S4: Fig. S6; Appendix S3: Table S2), which support the IGP hypothesis for spiders only. Hence, on average, birds reduced the total abundance of spiders by $57 \%$ (SE: $\pm 21 \%$ ). Conversely, and contrary to the general trends observed in other studies, herbivores did not significantly differ in total abundance between treatments (exclosure: $89 \pm 19.1$, open: $84.9 \pm 13.1$, shade: $85.6 \pm 16$ ), indicating an absence of bird and shade effects. This could be due to cascading effects of IGP on herbivores and/or to the presence of plant specific herbivore species in these communities (EFS).

\section{Effects of birds on arthropod species composition}

In the pRDA of arthropod species composition with treatments as predictors (Fig. 2a; Appendix S4: Fig. S7), only the first constrained component representing differences between exclosure and control treatments was significant (Appendix S3: Table S3). The constrained variance represented $2 \%$ of the total variance (adjusted $R^{2}=0.005$ ). Both intermediate predators and herbivores contained species that were negatively affected by birds (i.e., near the exclosure centroid in Fig. 2a and with negative B-scores in Fig. 2b), species that were neutrally affected by birds (i.e., at mid distance between all three centroids in Fig. 2a and with B-scores near zero in Fig. 2b), and species that were positively affected by birds (i.e., near the open and shade centroids in Fig. 2a and with positive B-scores in Fig. 2b).

Nevertheless, a significant majority of the intermediate predator species were negatively affected by birds, representing $63 \%$ of the total abundance of intermediate predators ( $91 \%$ of the abundance of which were spiders whereas predatory insect species were more often neutrally or positively affected by birds; Fig. 2b; Appendix S4: Figs. S7-S10), again consistent with IGP by birds on spiders. Herbivores comprised more equal proportions of species negatively, neutrally and positively affected by birds compared to intermediate predators (Fig. 2; Appendix S4: Figs. S7-S10). This suggests that IGP (bird control of spiders) could have benefited certain herbivore species (those positively or neutrally affected by birds), but not others (those negatively affected by birds). Therefore, on average, the effect of birds on herbivore species was not different from zero (Fig. 2b), again contradicting our hypothesis that birds should control overall herbivore abundance.

Shade effects on arthropod species were likely weak compared to bird effect, since the second constrained axis separating open and shade centroids was only marginally significant (Fig. 2a; Appendix S3: Table S3), which confirms the results observed on total arthropod abundance (Fig. 1). However, it cannot be excluded that part of the bird effect observed on some species was explained by the effect of shade.

\section{Plant specificity, bird effect and predation pressure}

After forward selection, plant species composition significantly explained arthropod species composition along seven significant principal components ("PC1", "PC6",,PC4", "PC16", "PC3", "PC108" and "PC7", which represented 


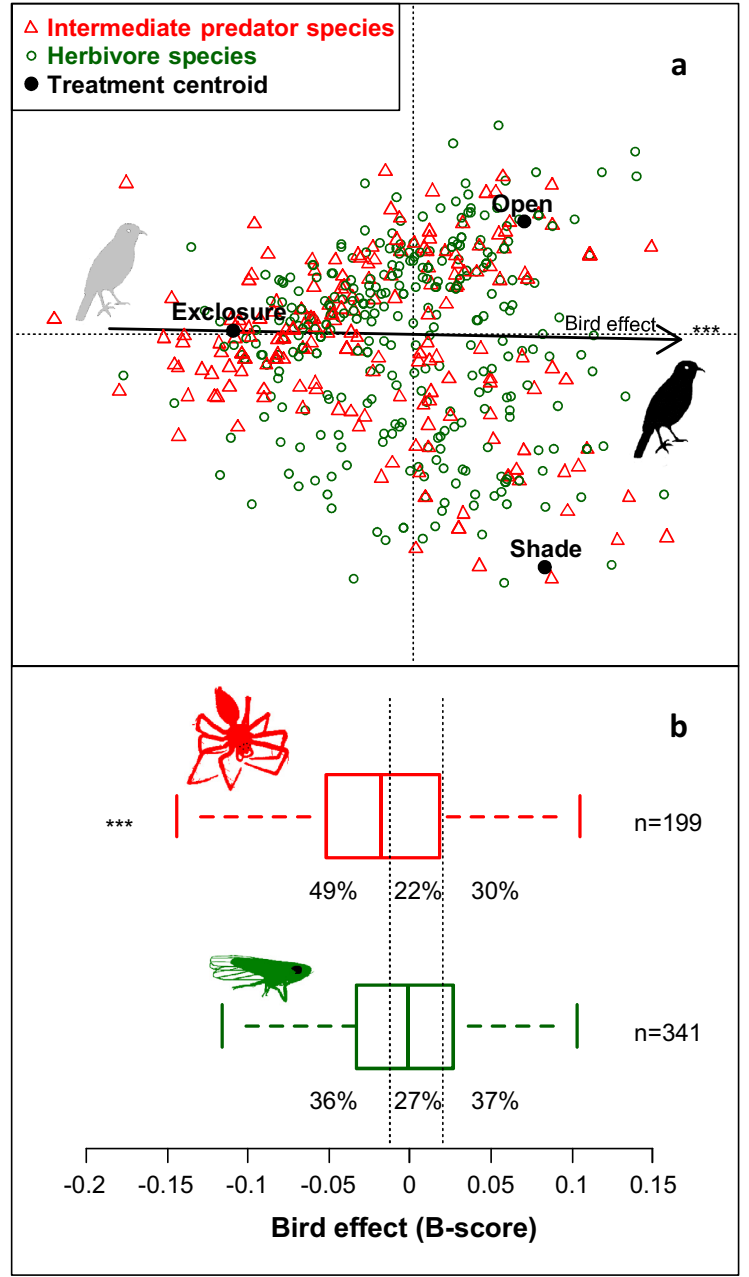

FIG. 2. Effect of birds on intermediate predators and herbivores. (a) Arthropod species scores and treatment centroids in the pRDA with treatments as predictors, for the constrained components 1 (horizontal axis) and 2 (vertical axis). Significance determined by permutational ANOVA (detailed version, Appendix S4: Fig. S7). (b) Bird effect on arthropod species (B-scores; median, quartiles, range) for intermediate predators (top) and herbivores (bottom) determined from the pRDA on arthropods with treatments as predictor. Significance of the deviation from zero determined with one-sampled $t$-test. The vertical dotted lines represents the negatively/neutrally/positively affected limits. Percentages of species belonging to each group are given. ${ }^{* * *} P \leq 0.001$.

$27.4 \%$ of the total variance in plant species composition). ANOVA with permutations determined that the first four constrained components of the pRDA were very significant $(P \leq 0.001$; Appendix S4: Fig. S11; Appendix S3: Table S4), which indicates strong correlations between some arthropod and plant species. However, as the constrained components 5 and 6 were also very close to significance, the P-scores (i.e., plant specificity estimates) were calculated based on the sixfirst components (representing $88.4 \%$ of the constrained variance; Appendix S3: Table S4). The constrained variance represented $6.8 \%$ of the total variance (adjusted $R^{2}=0.014$ ). The estimation of plant specificity was relatively robust to the number of variables included in the analysis (see discussion in Appendix S2; details of P-scores in Appendix S4: Figs. S12, S13).

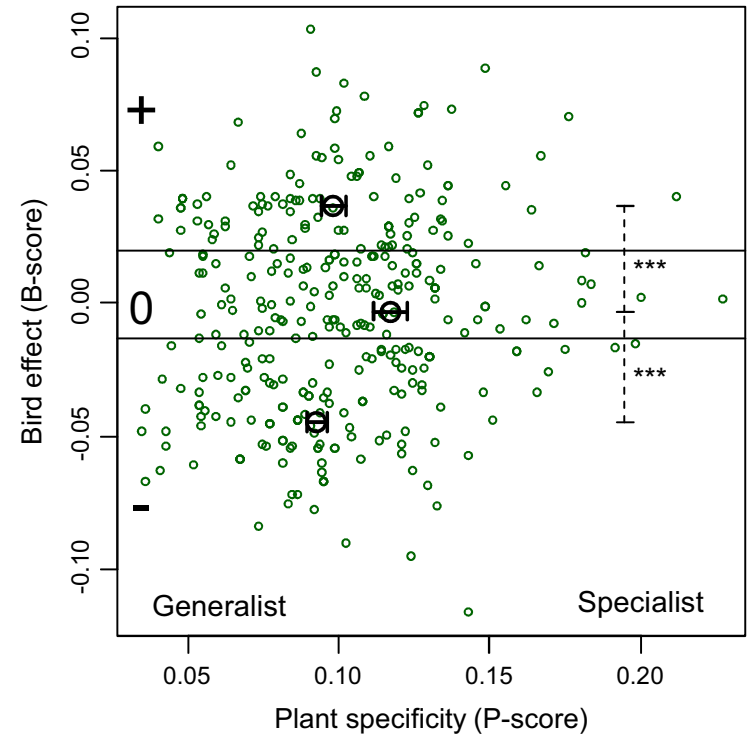

FIG. 3. Effect of birds on each herbivore species abundance (i.e., B-scores) according to their plant specificity (i.e., P-scores); B-scores determined from $\mathrm{pRDA}$ on arthropods with treatments as predictor; P-scores determined from pRDA on arthropods with plant species composition as predictors. Small dots represent raw data; large dots with bars represent estimated mean P-scores \pm SE determined by a linear mixed model (LMM) for herbivore species categorized by bird effect on them (-: negative; 0 : neutral; +: positive). Vertical dotted bars to the right show the significance of the REML $t$-tests with Satterthwaite's approximation in the LMM: ${ }^{* * *} P \leq 0.001$. Only significant comparisons are shown.

When looking at the effect of birds on the herbivore species (i.e., B-scores) according to their host-plant specificity (i.e., P-scores), a significant pattern could be detected (Fig. 3; Appendix S3: Table S5). Specifically, the herbivore species that were either negatively or positively affected by birds were less specialized to plants (estimated mean P-score $\pm \mathrm{SE}$ : $0.093 \pm 0.003$ and $0.098 \pm 0.004$, respectively) than the herbivore species neutrally affected by birds $(0.117 \pm 0.005)$ (Fig. 3; Appendix S3: Table S5). This suggests that plant generalists may have been more vulnerable to birds and/or spiders than plant specialists, and is consistent with the idea that EFS provided by plants protects plant specialists from both birds and spiders. However, a lot of variation in B-scores not attributable to P-scores (i.e., noise) was visible, and the detected pattern showed some variation across herbivore orders (Appendix S4: Fig. S14; Appendix S3: Table S5).

\section{DisCUSSION}

We have shown that birds preyed on intermediate predators, especially spiders, in this diverse ecosystem (Figs. 1, 4), but contrary to expectations based on earlier studies, overall herbivore abundance was unaffected by birds. However, when herbivore species were considered separately, the effects of birds ranged from negative, through neutral, to positive. This could be interpreted in terms of their relative susceptibility to predation by birds and spiders, as well as their specificity to plants. Herbivores species that were either negatively or positively affected by birds were less specialized to plants than species that were neutrally affected by birds. Although this 


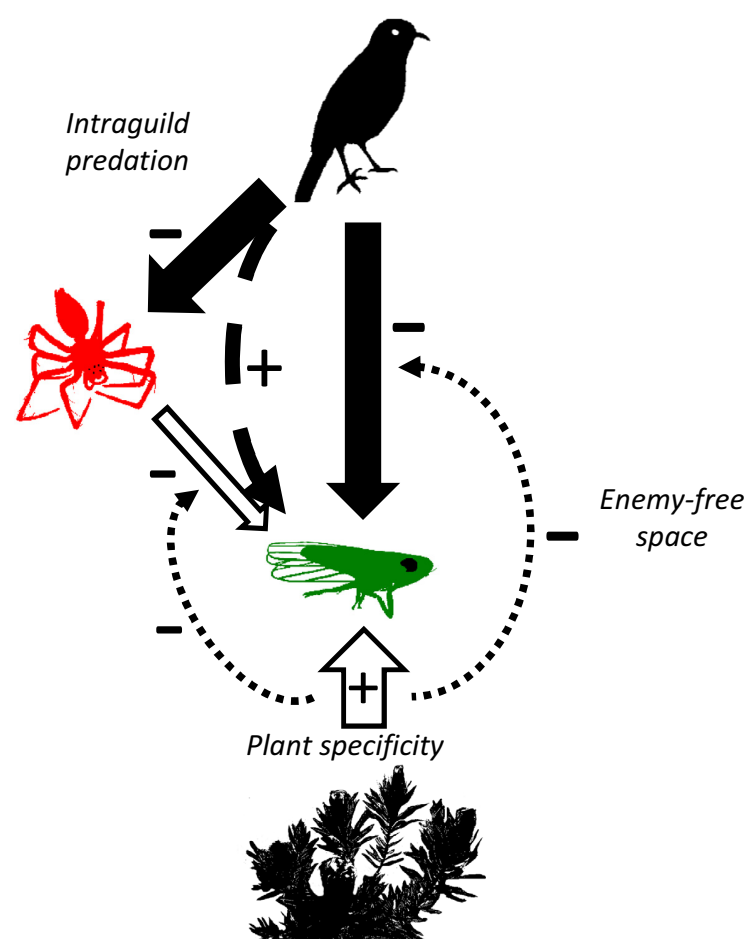

FIG. 4. Proposed model for the effect of birds, intermediate predators and plants on the average herbivore species. Plain block arrows represent direct negative effects of birds on intermediate predator and herbivore species determined from the exclosure experiment. The dash-lined arrow represents indirect positive effect of birds on herbivore species. Empty block arrows represent interactions determined with indirect methods: descending arrow represents presumed negative effect of intermediate predators on herbivores and ascending arrow represents presumed positive effects of plant specificity on herbivores. The dotted-lined arrow represent potential enemy-free space provided by plants to the most plant-specialized herbivore species. Arrow width represent the potential importance of the interaction.

pattern explains a small proportion of the observed variance, it supports the hypothesis of a higher vulnerability of plantgeneralist herbivores to predators (because plants do not provide enemy free space, EFS). This higher vulnerability would in turn result in a net negative effect of birds for the species mainly consumed by birds, but a net positive effect of birds for the species mainly consumed by spiders (because IGP releases these herbivore species; Fig. 4). Species that are equally affected by birds and spiders could be neutrally affected by birds (if IGP compensates for bird predation). However, as there was a tendency for the herbivore species that were neutrally affected by birds to be plant specialists, this could indicate that some of these species benefited from low vulnerability to both birds and spiders because of EFS provided by plants (Fig. 4). Nevertheless, because of uncertainties in the estimation of plant specificity in our study (see discussion in Appendix S2), the importance of EFS in these communities could not be accurately estimated. In particular, we lacked independent information on the diet breadth and species identity of most of our morpho-species.

In our study, release from predation by birds was a likely explanation for the much greater abundance of spiders in the exclosure cages. Spiders are known to be an important component of the diet of insectivorous birds (Gunnarsson 2007).
However, very little information exists on the feeding regime of the bird species present in our study site. A majority of them belonged to the Promeropidae and Nectariniidae, two families that feed on nectar and arthropods (Williams 1955). Members of the latter family are well-known for feeding their chicks with spiders and insects during the breeding season (Maher 1996). An alternative hypothesis to bird predation could be that the exclosure structure itself could have offered more anchor points to web-building spiders. However, there were as many spiders in the shade control as in the open plots, and the specific spider species that were positively affected by bird exclusion included both web-building spiders (e.g., Theridiidae, Araneidae) and hunting spiders (e.g., Philodromidae, Clubionidae). The greater abundance of some herbivore species in the exclosure cages may also have led to increased spider numbers (bottom-up effect; Kagata and Ohgushi 2006). In addition, changes in abundances of some intermediate predators or herbivores in the exclosures could also be due to responses to different micro-environmental conditions (e.g., soil, plant structure, temperature, humidity; Gunnarsson 1990, Randlkofer et al. 2010). Interactions with other arthropods not considered in our study such as parasitoids and detritivores or other vertebrates which had access to the exclosures such as lizards could also have influenced them (Spiller and Schoener 1990).

Contrary to the general trend pointed out by Mooney et al. (2010), birds did not have an overall negative effect on herbivore abundance in our study. While some species were negatively affected by birds, the presence of species released by birds but also of plant specialists unaffected by predators possibly contributed to an overall neutral effect of birds on herbivore communities. This seems likely since one of the main hypothesis explaining the specialization of herbivorous insects to plants is predator avoidance, with plant specialists notably benefiting from greater crypsis or chemical defense (EFS; Bernays and Graham 1988, Singer et al. 2014). For example, in the region (Cape Floristic Region), the Cephalelini (Cicadellidae; Hemiptera) present morphological traits to mimic sheaths of the reed-like Restionaceae (Augustyn et al. 2013,2017 ) on which they feed and to which they are often specific at the species level (Augustyn et al. 2013, Kemp et al. 2017). In our study, one Cephalelini morpho-species in particular was identified as one of the most plant-specialized herbivores (Cephalelini.sp4; Appendix S4: Fig. S11), though other species were classified as generalists. The evolution of their particular morphology in association with their host plants likely provides protection against visual predators such as birds (Augustyn et al. 2017). However, such visual crypsis would not offer protection from spiders, as most of the spider species in our study were web-builders or nocturnal wanderers, therefore not relying on visual cues to hunt their prey. Nevertheless, it could be possible that such plant-specialized insects are also less palatable than generalists, as shown with caterpillars (Bernays 1989, Dyer 1995), which would therefore also make them less vulnerable to spiders. In addition, plant specialization can be associated with lower mobility (Bernays 1989, Singer et al. 2014), which could provide additional protection against web-building spiders.

The negative to positive effects of birds on herbivore species could suggest that some species were consumed preferentially by birds whereas others were mainly consumed by 
spiders. Although the absence of information on the ecology of these herbivores makes it difficult to explain these preferences, some hypotheses could be proposed. For instance, it could be possible that ant-tended insects, such as aphids, are protected from spiders but are vulnerable to birds (Mooney and Linhart 2006). Oppositely, cryptic herbivore species with no chemical defense could be undetected by birds but still consumed by spiders that rely on tactile cues.

It must also be noted that it is possible that a small part of the effect of the bird exclosure cages could be due to additional shading created by the structure itself or to other uncontrolled effects. For instance, the relative humidity or wind speed inside the complete exclusion cages could have differed from that under the shade roofs. Conversely, the shade roofs may have differed from the open treatment in unintended ways: Even though birds were frequently observed to pass beneath the shade roofs, their predation rate on arthropods could have been affected by the roofs (scarecrow effect) (Botha 2017).

\section{Conclusion}

We showed that birds can differently affect various herbivore species, and this could depend on the cascading effects of bird intraguild predation on spiders, but also on the specificity of herbivores to plants, that would provide enemy-free space. In the current context of threats on biodiversity posed by human-caused modifications (Myers et al. 2000, Pimm and Raven 2000, Estes et al. 2011), considering such multitrophic effects would be of paramount importance to correctly predict the consequences of the extirpation of top predators such as birds on herbivore communities. In fynbos, where herbivore communities are expected to be dominated by plant specialists (Kemp et al. 2017; the current study), the extirpation of birds would have no overall effect on herbivores collectively, but at the species level, they may be either positively or negatively affected.

\section{ACKNOWLEDGMENTS}

Spider (Araneae) identifications were provided by Ansie Dippenaar-Schoeman (ARC), and adult voucher specimen are kept at the National Collection of Arachnida (Pretoria). Some plant species were identified by John C. Manning, Christopher N. Cupido, Nicky G. Bergh and E.G.,H Oliver (SANBI). We thank Michael Stiller (ARC) for his identification of Cicadellidae based on photographs. We also thank Pieter Botha for his help during plant survey and students who provided help for arthropod sorting. Funding was provided by the National Geographic Committee for Research and Exploration (Grant 9563-14), South African National Research Foundation (Grant 91507) and Stellenbosch University. Arthropod and plant specimens were collected in accordance with the conditions set by Cape Nature (permit number: 0056-AAA041-00028). C.B. conducted field experiments, and collected and analyzed data. C.B., A.P., F.R. and C.H. wrote and revised the manuscript.

\section{Literature Cited}

Augustyn, W. J., B. Anderson, M. Stiller, and A. G. Ellis. 2013. Specialised host-use and phenophase tracking in restio leafhoppers (Cicadellidae: Cephalelini) in the Cape Floristic Region. Journal of Insect Conservation 17:1267-1274.

Augustyn, W. J., B. Anderson, J. F. van der Merwe, and A. G. Ellis. 2017. Spatial turnover in host-plant availability drives host-associated divergence in a South African leafhopper (Cephalelus uncinatus). BMC Evolutionary Biology 17:72.

Bates, D., M. Mächler, B. Bolker, and S. Walker. 2014. Fitting linear mixed-effects models using lme4. Journal of Statistical Software 67:1-48.

Bernays, E. A. 1989. Host range in phytophagous insects: the potential role of generalist predators. Evolutionary Ecology 3:299-311.

Bernays, E. A., and M. Graham. 1988. On the evolution of host specificity in phytophagous arthropods. Ecology 69:886-892.

Borcard, D., P. Legendre, and P. Drapeau. 1992. Partialling out the spatial component of ecological variation. Ecology 73:1045-1055.

Borcard, D., F. Gillet, and P. Legendre. 2011. Numerical ecology with R. Springer, Berlin, Germany.

Borer, E. T., E. W. Seabloom, J. B. Shurin, K. E. Anderson, C. A. Blanchette, B. Broitman, S. D. Cooper, and B. S. Halpern. 2005. What determines the strength of a trophic cascade? Ecology 86: 528-537.

Botha, P. W. 2017. The world without birds : an experimental test of the ecological significance of pollinating birds for plant communities. Dissertation. Stellenbosch University, Stellenbosch.

Dyer, L. A. 1995. Tasty generalists and nasty specialists? Antipredator mechanisms in tropical Lepidopteran larvae. Ecology 76: $1483-1496$.

Dyer, L. A., et al. 2007. Host specificity of Lepidoptera in tropical and temperate forests. Nature 448:696-699.

Estes, J. A., et al. 2011. Trophic downgrading of planet earth. Science 333:301-306.

Fraser, M. W. 1989. Short-term responses of birds to fire in old mountain fynbos. Ostrich 60:172-182.

Goldblatt, P., and J. C. Manning. 2002. Plant diversity of the Cape region of southern Africa. Annals of the Missouri Botanical Garden 89:281-302.

Grass, I., K. Lehmann, C. Thies, and T. Tscharntke. 2017. Insectivorous birds disrupt biological control of cereal aphids. Ecology 98:1583-1590.

Greeney, H. F., L. A. Dyer, and A. M. Smilanich. 2012. Feeding by lepidopteran larvae is dangerous: a review of caterpillars' chemical, physiological, morphological, and behavioral defenses against natural enemies. Invertebrate Survival Journal 9:7-34.

Gunnarsson, B. 1990. Vegetation structure and the abundance and size distribution of spruce-living spiders. Journal of Animal Ecology 59:743.

Gunnarsson, B. 2007. Bird predation on spiders: ecological mechanisms and evolutionary consequences. Journal of Arachnology 35:509-529.

Hairston, N. G., F. E. Smith, and L. B. Slobodkin. 1960. Community structure, population control, and competition. American Naturalist 94:421-425.

Kagata, H., and T. Ohgushi. 2006. Bottom-up trophic cascades and material transfer in terrestrial food webs. Ecological Research 21:26-34.

Kemp, J. E., H. P. Linder, and A. G. Ellis. 2017. Beta diversity of herbivorous insects is coupled to high species and phylogenetic turnover of plant communities across short spatial scales in the Cape Floristic Region. Journal of Biogeography 44:1813-1823.

Kuznetsova, A., P. Brockhoff, and R. Christensen. 2016. lmerTest: tests in linear mixed effects models. $\mathrm{R}$ package version 2.0-33. https://CRAN.R-project.org/package=lmerTest

Law, Y.-H., and J. A. Rosenheim. 2011. Effects of combining an intraguild predator with a cannibalistic intermediate predator on a species-level trophic cascade. Ecology 92:333-341.

Legendre, P., and E. D. Gallagher. 2001. Ecologically meaningful transformations for ordination of species data. Oecologia 129: 271-280.

Maher, W. 1996. Nestling food and feeding frequencies of the brown-backed honeyeater Ramsayornis modestus and the yellowbellied sunbird Nectarinia jugularis in Northern Queensland. Emu 96:17.

Manning, J. C. 2007. Field guide to fynbos. Struik Nature, Cape Town, South Africa. 
Mäntylä, E., T. Klemola, and T. Laaksonen. 2011. Birds help plants: a meta-analysis of top-down trophic cascades caused by avian predators. Oecologia 165:143-151.

Martin, E. A., B. Reineking, B. Seo, and I. Steffan-Dewenter. 2013 Natural enemy interactions constrain pest control in complex agricultural landscapes. Proceedings of the National Academy of Sciences of the United States of America 110:5534-5539.

Mooney, K. A., and Y. B. Linhart. 2006. Contrasting cascades: insectivorous birds increase pine but not parasitic mistletoe growth. Journal of Animal Ecology 75:350-357.

Mooney, K. A., D. S. Gruner, N. A. Barber, S. A. Van Bael, S. M. Philpott, and R. Greenberg. 2010. Interactions among predators and the cascading effects of vertebrate insectivores on arthropod communities and plants. Proceedings of the National Academy of Sciences of the United States of America 107:7335-7340.

Myers, N., R. A. Mittermeier, C. G. Mittermeier, G. A. B. da Fonseca, and J. Kent. 2000. Biodiversity hotspots for conservation priorities. Nature 403:853-858.

Novotný, V., Y. Basset, S. E. Miller, G. D. Weiblen, B. Bremer, L. Cizek, and P. Drozd. 2002. Low host specificity of herbivorous insects in a tropical forest. Nature 416:841-844.

Oksanen, J., F. G. Blanchet, R. Kindt, P. Legendre, P. R. Minchin, R. B. O'Hara, G. L. Simpson, P. Solymos, M. H. H. Stevens, and H. Wagner. 2017. vegan: community ecology package. R package version 2.4-3. https://CRAN.R-project.org/package=vegan

Pace, M. L., J. J. Cole, S. R. Carpenter, and J. F. Kitchell. 1999. Trophic cascades revealed in diverse ecosystems. Trends in Ecology \& Evolution 14:483-488.

Pimm, S. L., and P. Raven. 2000. Biodiversity: extinction by numbers. Nature 403:843-845.

Polis, G. A., and R. D. Holt. 1992. Intraguild predation: the dynamics of complex trophic interactions. Trends in Ecology \& Evolution 7:151-154.
R Core Team. 2017. R: a language and environment for statistical computing. R Foundation for Statistical Computing, Vienna, Austria. http://www.R-project.org/.

Randlkofer, B., E. Obermaier, M. Hilker, and T. Meiners. 2010. Vegetation complexity-The influence of plant species diversity and plant structures on plant chemical complexity and arthropods. Basic and Applied Ecology 11:383-395.

Scholtz, C. H., and E. Holm. 1985. Insects of southern Africa. Butterworths, Durban, South Africa.

Singer, M. S., I. H. Lichter-Marck, T. E. Farkas, E. Aaron, K. D. Whitney, and K. A. Mooney. 2014. Herbivore diet breadth mediates the cascading effects of carnivores in food webs. Proceedings of the National Academy of Sciences of the United States of America 111:9521-9526.

Spiller, D. A., and T. W. Schoener. 1990. Lizards reduce food consumption by spiders: mechanisms and consequences. Oecologia 83:150-161.

Swart, R. C., J. S. Pryke, and F. Roets. 2017. Optimising the sampling of foliage arthropods from scrubland vegetation for biodiversity studies. African Entomology 25:164-174.

Vance-Chalcraft, H. D., J. A. Rosenheim, J. R. Vonesh, C. W. Osenberg, and A. Sih. 2007. The influence of intraguild predation on prey suppression and prey release: a meta-analysis. Ecology 88:2689-2696.

Vencl, F. V., F. Nogueira-De-Sá, B. J. Allen, D. M. Windsor, and D. J. Futuyma. 2005. Dietary specialization influences the efficacy of larval tortoise beetle shield defenses. Oecologia 145:404414.

Vidal, M. C., and S. M. Murphy. 2018. Bottom-up vs. top-down effects on terrestrial insect herbivores: a meta-analysis. Ecology Letters 21:138-150.

Williams, J. G. 1955. A systematic revision and natural history of the shining sunbird of Africa. Condor 57:249-262.

\section{SUPPORTING INFORMATION}

Additional supporting information may be found in the online version of this article at http://onlinelibrary.wiley.com/doi/10.1002/ecy. 2377/suppinfo

\section{Data Availability}

Data associated with this study are available in the Dryad Digital Repository: https://doi.org/10.5061/dryad.6k939m4. 